\title{
Inhibitory effects of vancomycin and fosfomycin on methicillin-resistant Staphylococcus aureus from antibiotic- impregnated articulating cement spacers
}

\section{Yuenyongviwat, \\ N. Ingviya, \\ P. Pathaburee, \\ B. Tangtrakulwanich}

Prince of Songkla

University, Songkhla, Thailand

- V. Yuenyongviwat, MD Orthopaedic Surgeon,

Department of Orthopaedic

Surgery and Physical Medicine,

Faculty of Medicine,

N. Ingviya, BSc, Scientist,

Microbiology Unit, Department of

Pathology, Faculty of Medicine,

- P. Pathaburee, MS, Scientist,

Scientific Equipment Center,

- B. Tangtrakulwanich, MD,

PhD, Orthopaedic Surgeon,

Department of Orthopaedic

Surgery and Physical Medicine,

Faculty of Medicine, Prince of

Songkla University, Songkhla,

90110, Thailand.

Correspondence should be sent to Dr V Yuenyongviwat; e-mail:

varahortho@gmail.com

doi:10.1302/2046-3758.63.2000639

Bone Joint Res 2017;6:132-136.

Received: 17 November 2015;

Accepted: 16 January 2017

\section{Objectives}

Vancomycin and fosfomycin are antibiotics commonly used to treat methicillin-resistant Staphylococcus aureus (MRSA) infection. This study compares the in vitro inhibitory effects against MRSA of articulating cement spacers impregnated with either vancomycin or fosfomycin.

\section{Methods}

Vancomycin-impregnated articulating cement spacers and fosfomycin-impregnated articulating cement spacers were immersed in sterile phosphate-buffered saline (PBS) solutions and then incubated. Samples were collected for bioactivity evaluation. The aliquots were tested for MRSA inhibition with the disc diffusion method, and the inhibition zone diameters were measured. The inhibition zone differences were evaluated using the Wilcoxon Rank Sum Test.

\section{Results}

The vancomycin group had significantly larger inhibition zones than the fosfomycin group from day three through to completion of the fourth week of incubation $(p<0.001)$. The vancomycin group exhibited a MRSA inhibition zone up to four weeks but the fosfomycin group showed an inhibition zone for only three days and after that did not show the the potential to inhibit MRSA.

\section{Conclusion}

This in vitro study found that the inhibitory effect of vancomycin-impregnated articulating cement spacers against MRSA outperformed fosfomycin-impregnated articulating cement spacers. Further comparing our results to other published reports suggests there might be a limitation of the disc diffusion bioassay to show a large inhibitory zone in a high concentration of a highly soluble antibiotic.

Cite this article: Bone Joint Res 2017;6:132-136

Keywords: Cement spacer, Vancomycin, Fosfomycin, TKA, MRSA

\section{Article focus}

- This study investigated the in vitro inhibitory effect against methicillin-resistant Staphylococcus aureus (MRSA) of vancomycin and fosfomycin in articulating cement spacers.

\section{Key messages}

- Vancomycin-impregnated articulating cement spacers showed a significantly better inhibitory effect against MRSA than cement spacers impregnated with fosfomycin.

- The vancomycin group showed an inhibitory effect for four weeks but the fosfomycin group showed an inhibitory effect for only three days.

\section{Strengths and limitations}

- Strength: This study is the first in vitro study to compare the inhibitory effects against MRSA of articulating cement spacers impregnated with either vancomycin or fosfomycin.

- Limitation: This study is an in vitro study. Therefore, further in vivo studies are necessary to assess the inhibitory effects against MRSA. 


\section{Introduction}

Infected total knee arthroplasty (TKA) is a devastating complication that can manifest with knee pain and decreased range of movement. The treatment protocol for an infected TKA patient depends on the severity and duration of the infection. In patients who cannot retain a prosthesis due to chronic infection, two-stage implantation is the benchmark for treatment of this condition. ${ }^{1-3}$

In the first stage of the two-stage re-implantation procedure, the surgeon needs to remove the original prosthesis, debride the infected tissue and insert an antibiotic cement spacer to fill the knee space. The spacer provides a high concentration of local antibiotic and maintains joint stability and joint space. ${ }^{4}$ After the first stage, the patient receives systemic antibiotics to eliminate infection. Finally, after the infection is eradicated, the second stage of the procedure is the insertion of a new prosthesis. ${ }^{5}$

An antibiotic-impregnated articulating cement spacer (mobile spacer), has advantages over a static spacer or block spacer. It maintains a greater range of movement, and promotes higher functional knee scores after the second-stage re-implantation by minimising soft-tissue contraction. 6,7

Staphylococcus aureus is the most common organism that causes infected TKA and methicillin-resistant Staphylococcus aureus (MRSA) periprosthetic infections have a high treatment failure rate. ${ }^{8-11}$ Commonly used in the treatment of MRSA, vancomycin and fosfomycin are antibiotics which inhibit bacterial cell wall synthesis. ${ }^{12-14}$ In the form of a local antibiotic delivery system, vancomycin and fosfomycin antibiotic-impregnated beads were reported to have good effectiveness in controlling MRSA. ${ }^{15,16}$ In an in vitro study, Stevens et al ${ }^{17}$ reported that a vancomycin articulated spacer could inhibit Bacillus subtilis. However, to our knowledge, there are no in vitro studies reporting on the effectiveness of articulating cement spacers in inhibiting MRSA using vancomycin or fosfomycin.

This study investigated the in vitro inhibitory effect of vancomycin and fosfomycin in articulating cement spacers.

\section{Materials and Methods}

Two groups of antibiotic-impregnated articulating cement spacers were prepared: a vancomycin group and a fosfomycin group. Five cement spacers were used in each group.

Antibiotic-impregnated articulating cement spacer preparation. Five pairs of vancomycin-impregnated articulating cement spacers were prepared in the operating room under aseptic techniques. Each pair consisted of two parts: the femoral and tibial components (Fig. 1). A total of $40 \mathrm{~g}$ of methyl methacrylate polymer (Palacos R bone cement; Heraeus Kulzer $\mathrm{GmbH}$, Wehrheim, Germany)

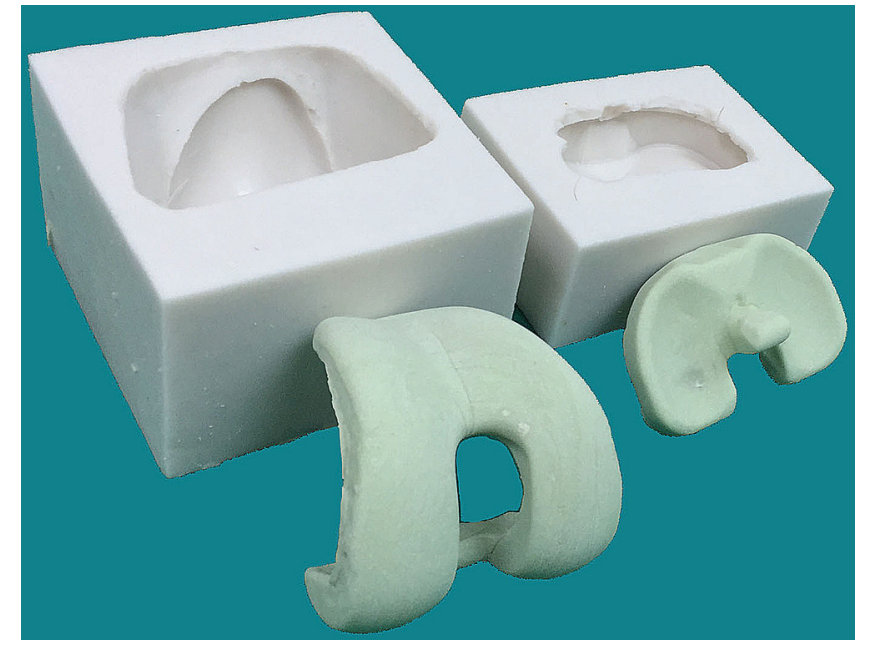

Fig. 1

Antibiotic-impregnated articulating cement spacers and silicone molds.

was mixed with $4 \mathrm{~g}$ of vancomycin powder (Vancogen; Alkem Laboratories Ltd, Daman, India) in a sterile bowl for five minutes. There were four mixing steps. Added together and mixed were10 g of methyl methacrylate polymer and $1 \mathrm{~g}$ of antibiotic. This was repeated until the final desired weights were reached. After mixing the antibiotic powder with the polymer, a liquid monomer was added. The mixture was then placed in an articulate spacer mould in the dough stage to create the uniform articulating cement spacers. After the cement had set, the spacers were removed from the moulds and stored in sterile bags for one hour before evaluating the inhibitory effect against MRSA. Five pairs of fosfomycinimpregnated articulating cement spacers were prepared following the same steps as for the vancomycin group but using a total of $4 \mathrm{~g}$ of fosfomycin powder (Fosmicin, Meiji Seika Kaisha Ltd, Tokyo, Japan) instead of vancomycin. The volumes of our femoral articulating cement spacers and tibial articulating cement spacers were measured by the Archimedes water displacement method. ${ }^{18}$ The volumes of the femoral articulating cement spacer and the tibial articulating cement spacer were $34 \mathrm{~cm}^{3}$ and $14 \mathrm{~cm}^{3}$, respectively.

Each set of vancomycin- and fosfomycin-impregnated articulating cement spacers were placed in sterile $600 \mathrm{~mL}$ beakers. The beakers were filled with $200 \mathrm{~mL}$ of sterile phosphate-buffered saline (PBS) solution pH 7.4 (SigmaAldrich Corp., St. Louis, Missouri) to fully immerse the cement spacers. The beakers were covered with sterile covers and placed in an incubator at $37^{\circ} \mathrm{C}$ for six weeks. Aliquots were collected and the remaining PBS solutions were discarded every 24 hours during the six weeks. Each set of cement spacers was then placed in fresh beakers with a new $200 \mathrm{~mL}$ sterile PBS solution. Samples from days one, three, five, seven, $14,21,28,35$, and 42 were evaluated for bioactivity. Samples from each beaker were 
Table I. Median (range) MRSA inhibition zones ( $\mathrm{mm}$ )

\begin{tabular}{llll}
\hline $\begin{array}{l}\text { Sample } \\
\text { (day) }\end{array}$ & Group & Fosfomycin & $\begin{array}{l}\text { p-value } \\
\text { (Wilcoxon } \\
\text { rank-sum } \\
\text { test) }\end{array}$ \\
\cline { 2 - 4 } & Vancomycin & $10.3(7.36$ to 15.24$)$ & 0.225 \\
\hline 1 & $12.31(11.93$ to 12.53$)$ & $0(0$ to 1.22$)$ & $<0.001$ \\
3 & $6.17(5.86$ to 6.79$)$ & $0(0$ to 0$)$ & $<0.001$ \\
5 & $5.06(4.75$ to 5.67$)$ & $0(0$ to 0$)$ & $<0.001$ \\
7 & $4.43(4.24$ to 4.91$)$ & $0(0$ to 0$)$ & $<0.001$ \\
14 & $2.5(1.99$ to 3.08$)$ & $0(0$ to 0$)$ & $<0.001$ \\
21 & $1.79(1.53$ to 2.09$)$ & $0(0$ to 0$)$ & 1 \\
28 & $0.89(0$ to 1.33$)$ & $0(0$ to 0$)$ & 1 \\
35 & $0(0$ to 0$)$ & $0(0$ to 0$)$ & \\
42 & $0(0$ to 0$)$ & & 0.001 \\
\hline
\end{tabular}

The diameter of the sterile filter paper disc $(6 \mathrm{~mm})$ was subtracted from the total inhibition zone

sent to a microbiologist with tag numbers to blind the group samples.

Inhibition test. Samples from each beaker were tested for MRSA inhibition, using a disc diffusion bioassay. Each cement spacer sample was tested using the five-disc diffusion method, on five culture plates. Vancomycin- and fosfomycin-susceptible MRSA were available from the microorganism bank. Informed consent was obtained from the donors before the microorganisms were used in this study. The MRSA isolates were obtained from tissue samples which are routinely sent from operative treatment of orthopaedic infected patients for the required identification of the microorganisms. The MRSA species were confirmed at the time of testing in this study. The antimicrobial susceptibility test followed the Clinical and Laboratory Standards Institute 2013 procedures. ${ }^{19}$ Mueller-Hinton agar plates were inoculated with a suspension equivalent to McFarland standard 0.5 for Staphylococcus aureus by swabbing the agar surface with a sterile cotton swab. Blank sterile filter paper discs of $6 \mathrm{~mm}$ diameter (Macherey-Nagel $\mathrm{GmbH} \&$ Co., Düren, Germany) infused with $10 \mu \mathrm{L}$ of sample solution were placed in the middle of each culture plate. The plates were incubated at $35^{\circ} \mathrm{C}$ for 24 hours. After 24 hours, the inhibition zone diameters were measured in millimetres with a digital vernier caliper.

Statistical analysis. The analyses were performed with $\mathrm{R}$ version 3.1.0 software ( $R$ Foundation for statistical computing, Vienna, Austria). The inhibition zone distance differences were evaluated using the Wilcoxon rank-sum test. Statistical significance was assumed if $p<0.05$.

\section{Results}

Inhibition zones from both groups are shown in Table I. Both groups demonstrated large inhibition circular zones in the day one samples and no significant differences were found in the inhibition zones $(p=0.23)$. The inhibition zones of the vancomycin group gradually declined with later samples, and still had an inhibitory effect throughout the fourth week (Fig. 2). However, the fosfomycin group showed an inhibitory effect for only three days. The vancomycin group had significantly larger inhibition zones than the fosfomycin group from sample day three through to completion of the fourth week $(p<0.001)$.

\section{Discussion}

Antibiotic-impregnated cement spacers offer the benefit of increased local antibiotic concentration at the infection site, and a higher success rate of infection control. ${ }^{20,21}$

The mechanism of antibiotic release from the antibioticimpregnated articulating cement is initially from the surface of the spacer. The antibiotic is then released through the network of cracks and voids in the surface of the articulating cement spacer as abrasion occurs. ${ }^{22}$ Salih et al ${ }^{23}$ reported that multiple indentations of the cement spacers increased the surface area which increased the elution of the antibiotic from the cement spacers.

The antibiotic of choice for the preparation of the cement spacers depends on the susceptibility of the micro-organism that causes the infection. Staphylococcus species are the most common causative bacteria in infected TKA. ${ }^{9}$ Kourbatova et al ${ }^{24}$ reported data from January 2003 to January 2004 that community-associated methicillin-resistant Staphylococcus aureus periprosthetic joint infection had emerged as a clinical reality. In the case of MRSA periprosthetic joint infection, antibiotic cement spacers need to be effective to eradicate MRSA.

The antibiotic dose for mixing in the cement for the treatment of acute infections is $2 \mathrm{~g}$ to $8 \mathrm{~g}$ per $40 \mathrm{~g}$ of poly (methyl methacrylate) (PMMA) for a prolonged and effective release of the antibiotic. ${ }^{25,26,27}$ In our practice and in this study, we used $4 \mathrm{~g}$ per $40 \mathrm{~g}$ of PMMA to produce the antibiotic-impregnated cement spacers. Paz et $\mathrm{al}^{26}$ reported that a high dose of antibiotic affects the mechanical properties and strength of the cement spacer. However, the mechanical properties of the temporary cement spacer are not as important as the cement for the final implant for definite fixation. The recommended dose of antibiotic in the final implant should be less than $2 \mathrm{~g}$ per $40 \mathrm{~g}$ of PMMA. ${ }^{21}$

There are limited reports of in vitro studies of MRSA form of local control by vancomycin- or fosfomycinimpregnated cement. Chang et al $^{29}$ reported an in vitro study of antibiotic-loaded cement cylinders prepared from $4 \mathrm{~g}$ of vancomycin per $40 \mathrm{~g}$ of PMMA for inhibition of MRSA. The study showed a 21-day antibacterial effect. A study by Roth et al ${ }^{15}$ that added fosfomycin to RefobacinPalacos R cement (cement containing $0.8 \mathrm{~g}$ gentamicin and $0.5 \mathrm{~g}$ gentamicin sulfate) showed larger zones of MRSA inhibition from an elution test when compared with the control group (Refobacin-Palacos $\mathrm{R}$ without fosfomycin). The study showed that the fosfomycin and Refobacin-Palacos R effectively inhibited MRSA up to and including day ten. Buranapanitkit et al $^{16}$ reported the 


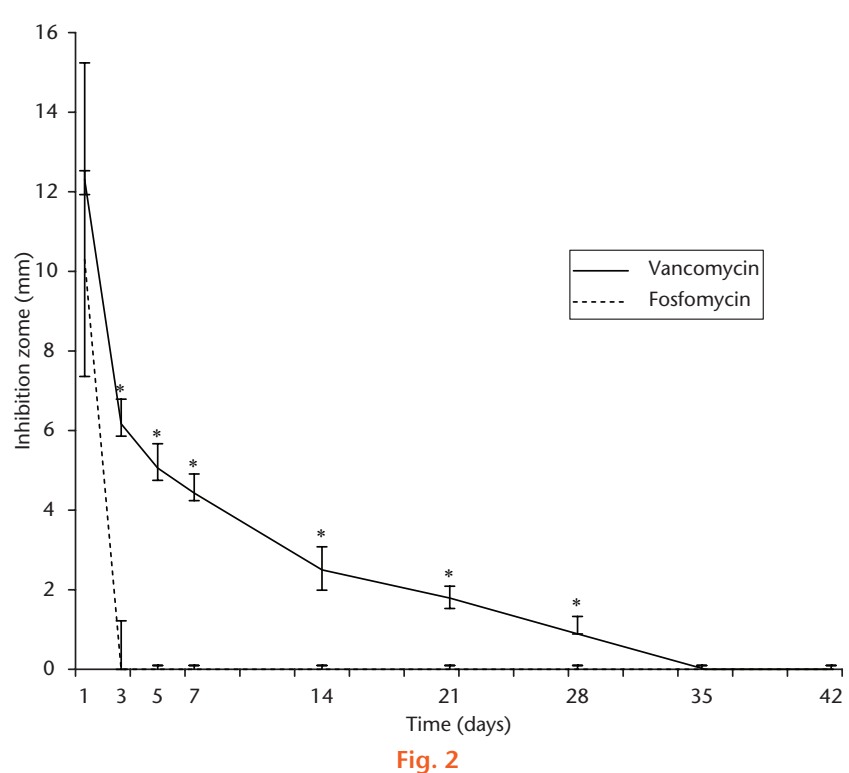

MRSA inhibition zones of vancomycin and fosfomycin from articulating cement spacers from the disc diffusion bioassay $\left({ }^{*} p<0.05\right.$, Wilcoxon ranksum test).

effectiveness of vancomycin and fosfomycin, antibiotic cement beads in inhibiting MRSA in vitro in the disc diffusion method. The study showed that the vancomycin cement beads could inhibit MRSA for three months and fosfomycin beads could inhibit MRSA for eight weeks. ${ }^{16}$

Compared with the study by Buranapanitkit et al, ${ }^{16}$ our results showed a shorter duration of MRSA inhibition in both vancomycin- and fosfomycin-impregnated articulating cement spacers. As our study completely immersed the cement spacers in the solution, the contact surface areas of the cement spacers and solution were higher than in the Buranapanitkit et al ${ }^{16}$ study, which placed the antibiotic cement beads directly on the culture plates. The antibiotic in the cement spacers in our study could elute more quickly than those in the Buranapanitkit et al ${ }^{16}$ study.

In our study, the fosfomycin group had large inhibition zones on the first day which then dropped rapidly after day one. The vancomycin group, however, had a gradual decrease in the inhibition zones. Lu et al ${ }^{30}$ reported that the molecular size of fosfomycin is smaller than that of vancomycin, and fosfomycin has a much higher solubility in water than that of vancomycin. These factors may be the reasons for the rapid reduction in the inhibition zones in the fosfomycin group compared with the vancomycin group. However, if the solubility of fosfomycin was the main factor, the inhibition zone of fosfomycin should have been larger than that of vancomycin on the first day. Our study found no difference in the inhibition zones between the two groups on the first day. We hypothesise, that this might be a limitation of the disc diffusion assay, in that it may be unable to show a large inhibitory zone in a high concentration of a highly soluble antibiotic. In order to prove this hypothesis, direct measurement of antibiotic concentrations and surface area calculation for each spacer could be done in future studies.

A limitation of this study was that we used only one strain of MRSA to test the inhibitory effect of antibioticimpregnated articulating cement spacers in a non-cyclical loading condition, in which the surface of the femoral and tibial components did not move against each other. Further study with more strains might find that fosfomycin may perform more effectively against other MRSA strains.

Our study showed that the cement spacers had an inhibitory effect for a period of time. Even though this antibiotic cement spacer has an inhibitory effect in vitro, this spacer may not be able to eradicate all bacteria in a clinical setting. Bacteria in prosthetic infections grow in their planktonic state and in biofilms, and will reactivate when the antibiotic level drops with time. Therefore, further studies in a controlled environment for the correct type, and dose, of antibiotic are needed.

This in vitro study found that the inhibitory effect of vancomycin-impregnated articulating cement spacers against MRSA outperformed fosfomycin-impregnated articulating cement spacers. Further comparing our results to other published reports, suggests there might be a limitation of the disc diffusion bioassay to show a large inhibitory zone in a high concentration of a highly soluble antibiotic.

\section{References}

1. Gooding CR, Masri BA, Duncan CP, et al. Durable infection control and function with the PROSTALAC spacer in two-stage revision for infected knee arthroplasty. Clin Orthop Relat Res 2011;469:985-993.

2. Castelli CC, Gotti V, Ferrari R. Two-stage treatment of infected total knee arthroplasty: two to thirteen year experience using an articulating preformed spacer. Int Orthop 2014;38:405-412.

3. Kuzyk PRT, Dhotar HS, Sternheim A, et al. Two-stage revision arthroplasty for management of chronic periprosthetic hip and knee infection: techniques, controversies, and outcomes. J Am Acad Orthop Surg 2014;22:153-164.

4. Romanò $\mathbf{C L}$, Gala L, Logoluso $\mathbf{N}$, et al. Two-stage revision of septic knee prosthesis with articulating knee spacers yields better infection eradication rate than one-stage or two-stage revision with static spacers. Knee Surg Sports Traumatol Arthrosc 2012;20:2445-2453

5. Bassetti M, Cadeo B, Villa G, et al. Current antibiotic management of prosthetic joint infections in Italy: the 'Udine strategy'. J Antimicrob Chemother 2014;69:i41-i45.

6. Chiang E-R, Su Y-P, Chen T-H, et al. Comparison of articulating and static spacers regarding infection with resistant organisms in total knee arthroplasty. Acta Orthop 2011;82:460-464

7. Fehring TK, Odum S, Calton TF, Mason JB. Articulating versus static spacers in revision total knee arthroplasty for sepsis. The Ranawat Award. Clin Orthop Relat Res 2000;380:9-16.

8. Schwarzkopf R, Oh D, Wright E, et al. Treatment Failure Among Infected Periprosthetic Patients at a Highly Specialized Revision TKA Referral Practice. Open Orthop J 2013;7:264-271.

9. Kaminski A, Citak M, Schildhauer TA, Fehmer T. Success rates for initial eradication of peri-prosthetic knee infection treated with a two-stage procedure. Ortop Traumatol Rehabil 2014;16:11-16.

10. Zürcher-Pfund L, Uçkay I, Legout L, et al. Pathogen-driven decision for implant retention in the management of infected total knee prostheses. Int Orthop 2013;37:1471-1475

11. Mittal Y, Fehring TK, Hanssen A, et al. Two-stage reimplantation for periprosthetic knee infection involving resistant organisms. J Bone Joint Surg [Am] 2007;89-A:1227-1231 
12. Rodvold KA, McConeghy KW. Methicillin-resistant Staphylococcus aureus therapy: past, present, and future. Clin Infect Dis 2014;58:S20-S27.

13. Poeppl W, Lingscheid T, Bernitzky $\mathbf{D}$, et al. Efficacy of fosfomycin compared to vancomycin in treatment of implant-associated chronic methicillin-resistant Staphylococcus aureus osteomyelitis in rats. Antimicrob Agents Chemother 2014;58: $5111-5116$

14. Sultan A, Rizvi $\mathbf{M}$, Khan $\mathbf{F}$, et al. Increasing antimicrobial resistance among uropathogens: is fosfomycin the answer? Urol Ann 2015;7:26-30

15. Roth KE, Krause B, Siegel E, et al. Liquid dextran does not increase the elution rate of different antibiotics from bone cement. Eur J Orthop Surg Traumatol 2015;25:83-89

16. Buranapanitkit B, Srinilta V, Ingviga N, et al. The efficacy of a hydroxyapatite composite as a biodegradable antibiotic delivery system. Clin Orthop Relat Res 2004:424:244-252.

17. Stevens CM, Tetsworth KD, Calhoun JH, Mader JT. An articulated antibiotic spacer used for infected total knee arthroplasty: a comparative in vitro elution study of Simplex and Palacos bone cements. J Orthop Res 2005;23:27-33.

18. Muller SD, Green SM, McCaskie AW. The dynamic volume changes of polymerising polymethyl methacrylate bone cement. Acta Orthop Scand 2002;73: 684-687.

19. Wayne P. Performance standards for antimicrobial susceptibility testing; twenty third informational supplement. Clinical and Laboratory Standards Institute. 2013.

20. Kim YS, Bae KC, Cho CH, et al. Two-stage revision using a modified articulating spacer in infected total knee arthroplasty. Knee Surg Relat Res 2013;25:180-185.

21. Meek RMD, Masri BA, Dunlop D, et al. Patient satisfaction and functional status after treatment of infection at the site of a total knee arthroplasty with use of the PROSTALAC articulating spacer. J Bone Joint Surg [Am] 2003;85-A:1888-1892.

22. Rogers BA, Middleton FR, Shearwood-Porter N, et al. Does cyclical loading affect the elution of antibiotics from articulating cement knee spacers? J Bone Joint Surg [Br] 2011;93-B:914-920.

23. Salih S, Paskins A, Nichol T, Smith T, Hamer A. The cement spacer with multiple indentations: increasing antibiotic elution using a cement spacer 'teabag'. Bone Joint J 2015;97-B:1519-1524.

24. Kourbatova EV, Halvosa JS, King MD, et al. Emergence of community-associated methicillin-resistant Staphylococcus aureus USA 300 clone as a cause of health care- associated infections among patients with prosthetic joint infections. Am J Infect Control 2005;33:385-391.

25. Bistolfi A, Massazza G, Verné E, et al. Antibiotic-loaded cement in orthopedic surgery: a review. ISRN Orthop 2011;2011:290851.

26. Paz E, Sanz-Ruiz P, Abenojar J, et al. Evaluation of Elution and Mechanical Properties of High-Dose Antibiotic-Loaded Bone Cement: Comparative "In Vitro" Study of the Influence of Vancomycin and Cefazolin. J Arthroplasty. 2015;30-8:1423-1429.

27. Frew NM, Cannon T, Nichol T, Smith TJ, Stockley I. Comparison of the elution properties of commercially available gentamicin and bone cement containing vancomycin with 'home-made' preparations. Bone Joint J 2017;99-B:73-77.

28. Springer BD, Lee GC, Osmon D, et al. Systemic safety of high-dose antibioticloaded cement spacers after resection of an infected total knee arthroplasty. Clin Orthop Relat Res 2004;427:47-51.

29. Chang Y, Chen W-C, Hsieh P-H, et al. In vitro activities of daptomycin-, vancomycinand teicoplanin-loaded polymethylmethacrylate against methicillin-susceptible, methicillin-resistant, and vancomycin-intermediate strains of Staphylococcus aureus. Antimicrob Agents Chemother 2011;55:5480-5484.

30. Lu CL, Liu CY, Huang YT, et al. Antimicrobial susceptibilities of commonly encountered bacterial isolates to fosfomycin determined by agar dilution and disk diffusion methods. Antimicrob Agents Chemother 2011;55:4295-4301.

Funding

The study was funded by Faculty of Medicine, Prince of Songkla University, Songkhla, Thailand.

Author Contributions

V. Yuenyongviwat: Corresponding author, study design, data collection, writing the paper.

N. Ingviya: Data collection.

- P. Pathaburee: Data collection.

B. Tangtrakulwanich: Study design, Writing the paper.

ICMJE Conflicts of Interest

None declared.

(c) 2017 Yuenyongviwat et al. This is an open-access article distributed under the terms of the Creative Commons Attributions licence (CC-BY-NC), which permits unrestricted use, distribution, and reproduction in any medium, but not for commercial gain, provided the original author and source are credited. 